\title{
Computational modeling of GMI effect in Co-based amorphous ribbons
}

Asli Ayten Kaya*

Dedicated to Professor Hari M Srivastava

"Correspondence:

aslitay@uludag.edu.tr

Physics Department, Faculty of Arts

and Sciences, Uludag University,

Gorukle Campus, Bursa, 16059

Turkey

\begin{abstract}
This paper presents a prediction of a giant magneto-impedance (GMI) effect on Co-based amorphous ribbons using an artificial neural network (ANN) approach based on a self-organizing feature map (SOFM). The input parameters included the compositions of $\mathrm{Fe}$ and $\mathrm{Co}$, ribbon width and magnetizing frequency. The output parameter was the GMI effect. The results show that the proposed model can be used for estimation of the GMl effect in the amorphous ribbons.
\end{abstract}

Keywords: giant magneto-impedance effect; neural network; modeling

\section{Introduction}

When a soft ferromagnetic conductor is subjected to an alternating current, a large change in the complex impedance of the conductor can be achieved upon applying a magnetic field. This phenomenon is known as the giant magneto-impedance (GMI) effect [1]. This effect has received increasing attention for its potential applications in highly sensitive magnetic sensors [2].

The ANN is simply a class of mathematical algorithms, since a network can be regarded essentially as a graphic notation for a large class of algorithms. Such algorithms produce solutions to a number of specific problems [3].

In this investigation, the GMI effects are modeled using self-organizing feature map (SOFM) and previous experimental data [4, 5] of amorphous ribbons made from $\mathrm{Co}_{70} \mathrm{Fe}_{5} \mathrm{Si}_{15} \mathrm{~B}_{10}$ and $\mathrm{Co}_{70.4} \mathrm{Fe}_{4.6} \mathrm{Si}_{15} \mathrm{~B}_{10}$ alloys.

\section{Experimental details}

\section{Self-organizing feature map (SOFM)}

Self-organizing feature maps (SOFM), also known as Kohonen maps or topographic maps, were first introduced by von der Malsburg (1973) and in its present form by Kohonen (1982). SOFM is a special neural network that accepts $N$-dimensional input vectors and maps them to the Kohonen layer, in which neurons are organized in an $L$-dimensional lattice (grid) representing the feature space. Such a lattice characterizes a relative position of neurons with regards to their neighbors, that is, their topological properties rather than exact geometric locations. In practice, dimensionality of the feature space is often restricted by its visualization aspect and typically is $L=1,2$ or 3 . The objective of the learning algorithm for the SOFM neural networks is formation of the feature map which cap- 
tures the essential characteristics of the $N$-dimensional input data and maps them on the typically 1-D or 2-D feature space [6].

During training, the weights are updated according to the formula:

$$
w_{i j}(t+1)=w_{i j}(t)+\eta(t)\left(u_{i}-w_{i j}\right)\left\|u_{i}-w_{i j}\right\| N(j, t)
$$

where $w_{i j}$ and $u^{i}$ are the $i$ th component of the weight vector $\mathbf{w}_{j}$ of the neuron $n_{j}$ and the pattern $\mathbf{u}^{\mathrm{k}}$ applied to the input layer respectively, $\eta(t)$ is the learning rate and $N(j, t)$ is the neighborhood function which is changing in time. The learning algorithm captures two essential aspects of the map formation, namely competition and cooperation between neurons of the output lattice. Competition determines the winning neuron $d_{\text {win }}$, whose weight vector is the one closest to the applied input vector. For this purpose, the input vector $\mathbf{u}$ is compared with each weight vector $\mathbf{w}_{j}$ from the weight matrix $\mathbf{W}$, and the index of the winning neuron $n_{\text {win }}$ is established considering the following formula:

$$
n_{\text {win }}=\operatorname{argmin}\left\|\mathbf{u}-\mathbf{w}_{j}\right\| .
$$

All neurons $n_{j}$ located in a topological neighborhood of the winning neurons $n_{\text {win }}$ will have their weights updated usually with strength $N(j)$ related to their distance $d(j)$ from the winning neuron, where $d(j)$ can be calculated using the formula

$$
d(j)=\left\|\operatorname{pos}\left(n_{j}\right)-\operatorname{pos}\left(n_{\text {win }}\right)\right\|,
$$

where $\operatorname{pos}(\cdot)$ is the position of the neuron in the lattice [6].

\section{SOFM training algorithm}

1. Assign small random values to weights $\mathbf{w}_{j}=\left[w_{1 j}, w_{2 j}, \ldots, w_{n j}\right]$;

2. Choose a vector $\mathbf{u}^{\mathrm{k}}$ from the training set and apply it as input;

3. Find the winning output node $n_{\text {win }}$ by the following criterion:

$$
n_{\text {win }}=\underset{j}{\operatorname{argmin}}\left\|\mathbf{u}-\mathbf{w}_{j}\right\|
$$

where $\|\cdot\|$ denotes the Euclidean norm and $\mathbf{w}_{j}$ is the weight vector connecting input nodes to the output node $j$;

4. Adjust the weight vectors according to the following update formula:

$$
w_{i j}(t+1)=w_{i j}(t)+\eta(t)\left(u_{i}-w_{i j}\right)\left(u_{i}-w_{i j}\right) N(j, t)
$$

where $w_{i j}$ is the $i$ th component of the weight vector $\mathbf{w}_{j}, \eta(t)$ is the learning rate and $N(j, t)$ is the neighborhood function;

5. Repeat Steps 2 through 4 until no significant changes occur in the weights [6].

\section{Developed ANN model}

Two different amorphous ribbons were used for the experimental verification of the proposed model. A total of 1,200 input vectors were obtained from the amorphous ribbons $[4,5]$. The developed neural network, which has five input neurons, one output neuron, 


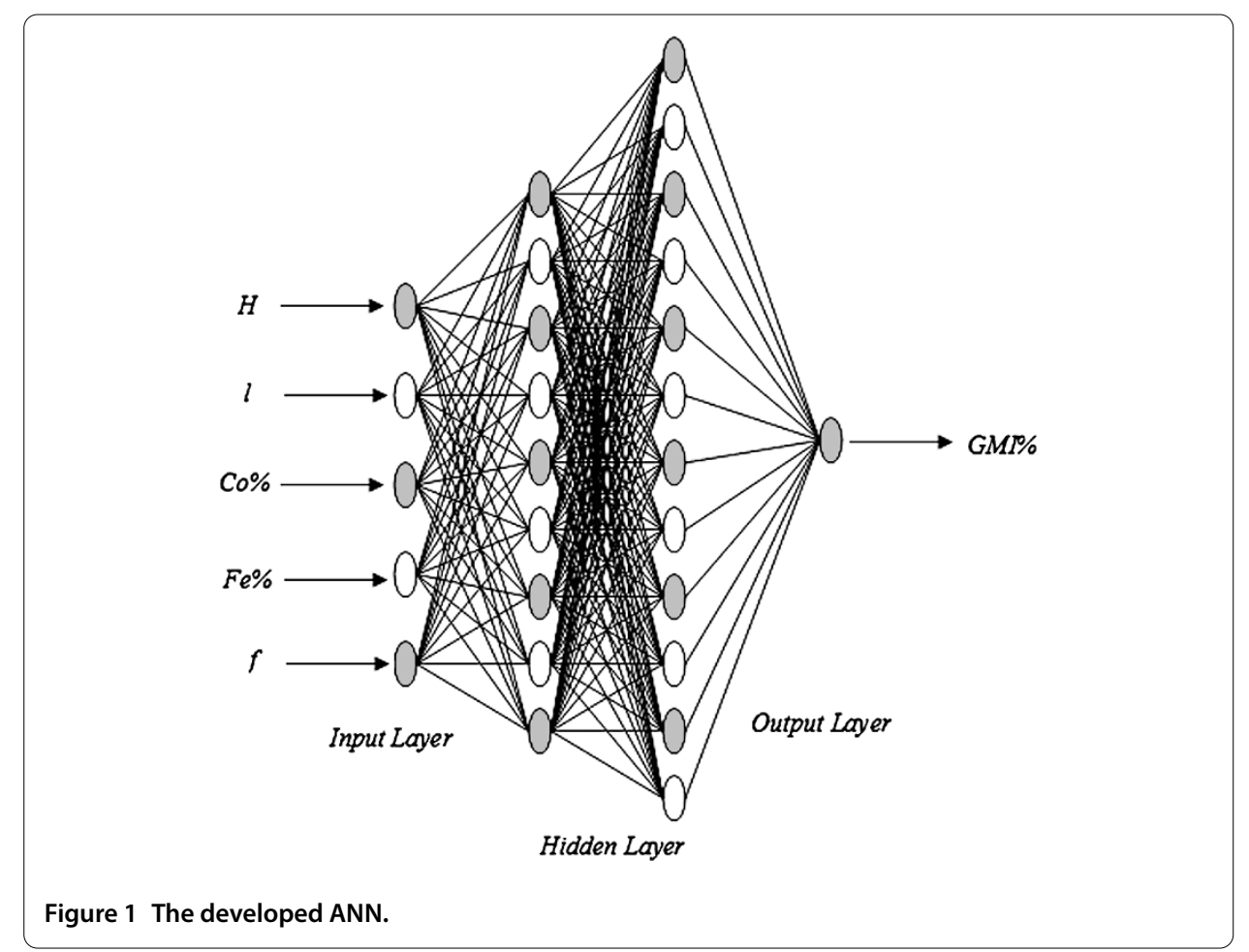

two hidden layers, nine and twelve neurons of hidden layers and full connectivity between neurons, was shown in Figure 1. The input parameters were magnetizing field $(H)$, ribbon width $(l)$, magnetizing frequency $(f)$, concentration of $\mathrm{Co}(\mathrm{Co} \%)$ and concentration of $\mathrm{Fe}$ (Fe\%). The output parameter was the GMI effect (GMI\%). The number of hidden layers, neurons in each hidden layer and training parameters were determined through trial and error to be optimal. After several trials, a better result was obtained from the network a four-layered network. In this network, the hyperbolic tangent function is used in the hidden and output layers. The number of epochs was 30,000 for training.

\section{Results and discussion}

In this study an attempt was made to predict the GMI effect of Co-based amorphous ribbons $\left(\mathrm{Co}_{70} \mathrm{Fe}_{5} \mathrm{Si}_{15} \mathrm{~B}_{10}\right.$ and $\left.\mathrm{Co}_{70.4} \mathrm{Fe}_{4.6} \mathrm{Si}_{15} \mathrm{~B}_{10}\right)$ using artificial neural networks. To achieve this goal, magnetizing field $(H)$, ribbon width $(l)$, magnetizing frequency $(f)$, concentration of $\mathrm{Co}(\mathrm{Co} \%)$ and concentration of $\mathrm{Fe}(\mathrm{Fe} \%)$ were used as the input of networks, and GMI\% data points were used as the output of these networks. Finally, network was achieved with the least cross validation error for Co-based amorphous ribbons.

As it is indicated in Figure 2, the predicted GMI data points using neural networks follow the experimental results in an appropriate manner. It is obvious that the predicted GMI\%, using neural networks and the experimental results, is found to be a perfect match for the trained data.

Figure 3 shows the GMI effect obtained from the prediction model and experimental data at 0.1-1 $\mathrm{MHz}$ for $\mathrm{Co}_{70} \mathrm{Fe}_{5} \mathrm{Si}_{15} \mathrm{~B}_{10}$ and $\mathrm{Co}_{70.4} \mathrm{Fe}_{4.6} \mathrm{Si}_{15} \mathrm{~B}_{10}$ amorphous ribbons which have $0.5-3 \mathrm{~cm}$ ribbon width. The GMI curves achieved from the ANN are in about $99 \%$ agreement with the experimental ones. 


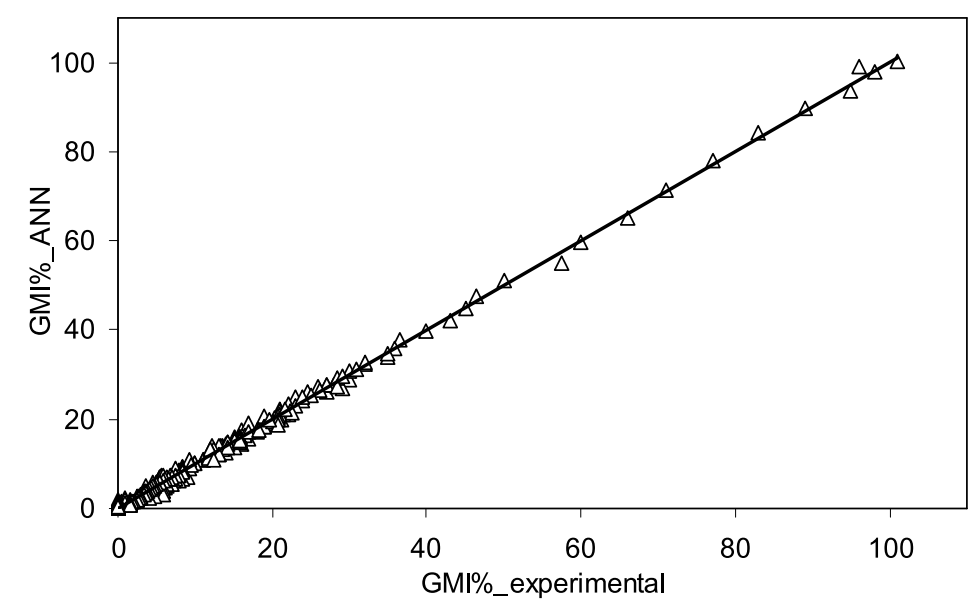

Figure 2 Plot of the measurement GMI\% values versus the network output.

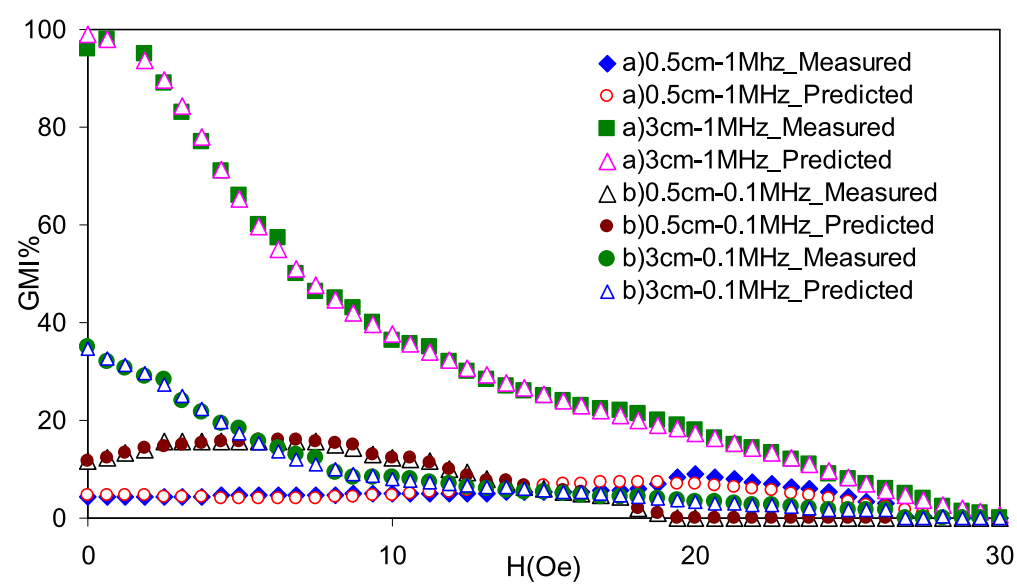

Figure 3 Comparisons of experimental data and predicted $\mathrm{GMI}$ effect in (a) $\mathrm{Co}_{70} \mathrm{Fe}_{5} \mathrm{Si}_{15} \mathrm{~B}_{10}$ and (b) $\mathrm{Co}_{70.4} \mathrm{Fe}_{4.6} \mathrm{Si}_{15} \mathrm{~B}_{10}$ ribbons.

All the tested samples in the range of training data have significant correlation coefficients. The ANN model was assessed with $1 \mathrm{~mm}$ width amorphous ribbons which are outside the range of the training data. Figure 4 shows good agreement with the experimental data; therefore, the ANN should be used for the prediction and modeling of GMI values.

These results show that the proposed model is potentially useful for sensor designers in predicting the GMI curves in cases when measurements may be time consuming.

\section{Conclusions}

In this study the proposed model was developed from experimental data for the Co-based amorphous ribbons. This study demonstrates the applicability and feasibility of artificial neural network models to predict the GMI effect for $\mathrm{Co}_{70} \mathrm{Fe}_{5} \mathrm{Si}_{15} \mathrm{~B}_{10}$ and $\mathrm{Co}_{70.4} \mathrm{Fe}_{4.6} \mathrm{Si}_{15} \mathrm{~B}_{10}$ amorphous ribbons which have different ribbon width such as $0.5,1$ and $3 \mathrm{~mm}$ and a frequency range of 0.1-1 MHz. The average correlation and prediction error were found to be $99 \%$ and $1 \%$ for the tested amorphous ribbons, respectively. These results show that the 


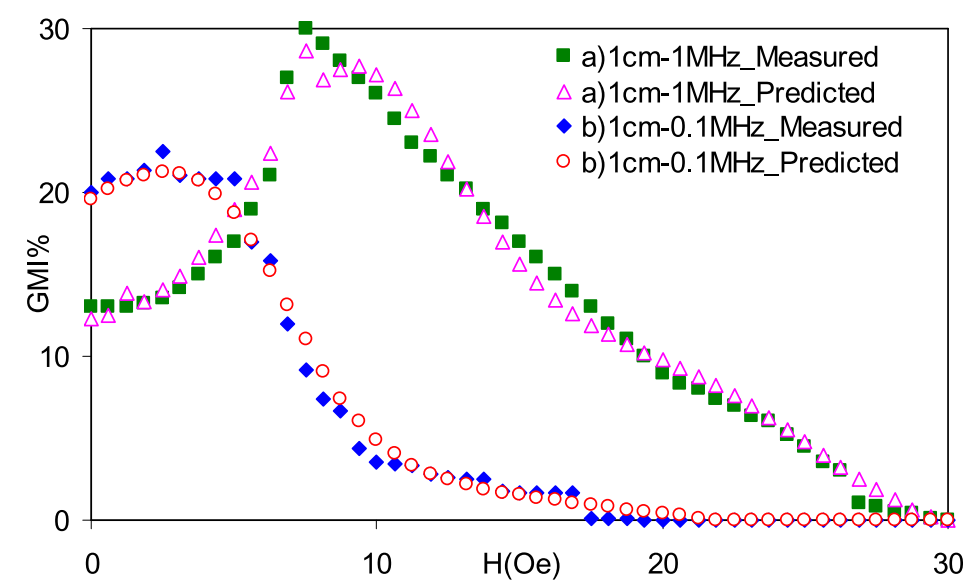

Figure 4 The variation of predicted and measured values for (a) $\mathrm{C}_{70} \mathrm{Fe}_{5} \mathrm{Si}_{15} \mathrm{~B}_{10}$ and (b) $\mathrm{Co}_{70.4} \mathrm{Fe}_{4.6} \mathrm{Si}_{15} \mathrm{~B}_{10}$ amorphous ribbons.

predicted values of these ribbons are in good agreement with the measured ones. Therefore, this model is appropriate for a researcher to evaluate the sensor performance before manufacture.

\section{Competing interests}

The author declares that they have no competing interests.

Received: 31 January 2013 Accepted: 28 May 2013 Published: 14 June 2013

\section{References}

1. Phan, MH: Giant magnetoimpedance materials: fundamentals and applications. Prog. Mater. Sci. 53, 323-420 (2008)

2. Gong, WY, Wu, ZM, Lin, H, Yang, XL, Zhao, Z: Longitudinally driven giant magneto-impedance effect enhancement by magneto-mechanical resonance. J. Magn. Magn. Mater. 320, 1553-1556 (2008)

3. Zurada, JM: Introduction to Artificial Neural Systems. West Publishing Company, Eagan (1992)

4. Goncalves, LAP, Soares, JM, Machado, FLA, de Azevedo, WM: GMI effect in the low magnetostrictive $\mathrm{CO}_{70} \mathrm{Fe}_{5} \mathrm{Si}_{15} \mathrm{~B}_{10}$ alloys. Physica B 384, 152-154 (2006)

5. Mendes, $\mathrm{KC}$, Machado, FLA: Enhanced GMl in ribbons of $\mathrm{CO}_{70.4} \mathrm{Fe}_{4.6} \mathrm{Si}_{15} \mathrm{~B}_{10}$ alloy. J. Magn. Magn. Mater. 177-181, 111-112 (1998)

6. Halici, U: Data Clustering and Self-Organizing Feature Maps. In: Artificial Neural Networks. EE543 Lecture Notes, ch.8, pp. 135-136. Metu Eee, Ankara (2004)

\section{Submit your manuscript to a SpringerOpen ${ }^{\circ}$ journal and benefit from:}

- Convenient online submission

- Rigorous peer review

- Immediate publication on acceptance

- Open access: articles freely available online

- High visibility within the field

- Retaining the copyright to your article 